\title{
Exploring dark current voltage characteristics of micromorph silicon tandem cells with computer simulations
}

\author{
A. Sturiale, ${ }^{1}$ Hongbo T. Li, ${ }^{2}$ J. K. Rath, ${ }^{2}$ R. E. I. Schropp, ${ }^{2}$ and F. A. Rubinelli ${ }^{1, a)}$ \\ ${ }^{1}$ INTEC, CONICET, Universidad Nacional del Litoral, Güemes 3450, 3000 Santa Fe, Argentina \\ ${ }^{2}$ Debye Institute, Utrecht University, P.O. Box 80000, 3508 TA Utrecht, The Netherlands
}

(Received 28 January 2009; accepted 13 May 2009; published online 2 July 2009)

\begin{abstract}
The transport mechanisms controlling the forward dark current-voltage characteristic of the silicon micromorph tandem solar cell were investigated with numerical modeling techniques. The dark current-voltage characteristics of the micromorph tandem structure at forward voltages show three regions: two with an exponential dependence and a third where the current grows more slowly with the applied voltage. In the first exponential region the current is entirely controlled by recombination through gap states of the top cell. In the second exponential region the current is controlled by the mixture of recombination through gap states of both the top and bottom cells and by free carrier diffusion along the $a-\mathrm{Si}: \mathrm{H}$ intrinsic layer. In the third region the onset of the electron space charge limited current on the $a-\mathrm{Si}: \mathrm{H}$ top cell can be observed along with some other mechanisms that are discussed in the paper. The high forward dark $J-V$ curve of the tandem cell can be used as diagnosis tool to quickly inspect the efficiency of the recombination junction in recombining the electron-hole pairs generated under illumination in the top and bottom cells. The dark current at high forward voltages is highly influenced by the amount of electron-hole pairs thermally generated inside the recombination junction. (C) 2009 American Institute of Physics. [DOI: 10.1063/1.3151691]
\end{abstract}

\section{INTRODUCTION}

In the technology of multijunction solar cells two or more single $p-i-n$ solar cells with different band gaps are stacked on top of each other. These complex structures can reach higher conversion efficiencies than single cells due to a more efficient utilization of the solar spectrum and to the improved collection of photogenerated carriers. ${ }^{1}$ In particular the concept of the micromorph tandem solar cell that combines amorphous hydrogenated silicon $(a-\mathrm{Si}: \mathrm{H})$ with microcrystalline silicon $(\mu c-\mathrm{Si})$ has become a promising strategy due to its simplicity in the design and to its good performance. ${ }^{2}$ The micromorph cell does not incorporate any silicon alloys in the absorber layers; it has the optimal bandgap combination for terrestrial applications, and it is quite stable to light induced degradation. ${ }^{3,4}$ Tandem solar cells are regularly characterized by their light current-voltage $(J-V)$ characteristics under AM1.5 illumination and by the spectral responses (SRs) under blue bias light and under red bias light. $^{2-4}$ To our knowledge the dark $J-V$ curves that have been widely used and studied in single solar cells have not been yet systematically explored in tandem solar cell structures to see if they can provide some useful information about the electrical quality of the device constituents.

Single $a$-Si: $\mathrm{H}$ based $p-i-n$ devices have been extensively studied by different research groups. ${ }^{5-8}$ The dark current $(J)$ at low forward biases increases exponentially with the applied voltage $(V)$ and it is controlled by recombination at the lowest voltages. The exponential dark $J-V$ characteristic is described by two parameters: the saturation current $J_{0}$ and the ideality factor $n$. The same current $J$ at high forward

\footnotetext{
${ }^{a)}$ Author to whom correspondence should be addressed. Electronic mail: frubinelli@santafe-conicet.gov.ar.
}

voltages increases more slowly with the applied voltage entering into the so-called space charge limited current (SCLC) regime. ${ }^{9,10}$ Dark $J-V$ characteristics of single $\mu c-\mathrm{Si}: \mathrm{H}$ based $p-i-n$ devices have also been studied in the literature but in less extent than in case of $a-\mathrm{Si}: \mathrm{H}$ based devices. ${ }^{11-14}$ The current at low forward voltages is a strong function of the silane $\left[\mathrm{SiH}_{4}\right]$ concentration (SC), defined as $\mathrm{SC}$ $=\left[\mathrm{SiH}_{4}\right] /\left(\left[\mathrm{SiH}_{4}\right]+\left[\mathrm{H}_{2}\right]\right)$, used in the gas phase to deposit in the intrinsic layer, the current being lower for higher values of SC. ${ }^{12,13}$ The current at low forward voltages for these devices is always higher than in $a-\mathrm{Si}: \mathrm{H}$ based $p-i-n$ devices due to the lower band gap of $\mu c-\mathrm{Si}: \mathrm{H}$ (Refs. 12-14) that strongly favors recombination. The high forward regime is claimed to be limited by series resistance according to the literature $^{11,12}$ but contact effects could also limit the current. $^{14}$

In this paper we explore using computer simulations the transport mechanisms existing in the dark $J-V$ of micromorph silicon tandem cells. We briefly revise the physics behind the dark $J-V$ characteristic curves of the three single structures present in the tandem: the $a-\mathrm{Si}: \mathrm{H}$ and the $\mu c-\mathrm{Si}: \mathrm{H}$ based $p-i-n$ devices and the $n-i-p$ tunnel recombination junction (TRJ). Using this knowledge we ponder the contribution of each of the mechanisms present in single devices in defining to the final shape of the dark $J-V$ of the tandem cell. Finally we match the experimental dark $J-V$ characteristics of one micromorph device.

Our simulations were performed with the computer code D-AMPS, ${ }^{15}$ an updated version of the program AMPS (Analysis of Microelectronic and Photonic Devices) developed at The Pennsylvania State University, University Park, USA. ${ }^{16}$ The letter D stands for new developments that were implemented by the last author of this manuscript. They refer to 
TABLE I. List of the key electric parameters used in the intrinsic HWCVD $a$-Si:H and $\mu c-\mathrm{Si}: \mathrm{H}$ layers. The meaning of the symbols is as follows: $E_{G}$ is the mobility gap in eV, DB is the global density midgap enclosed by the three Gaussians in $\mathrm{cm}^{-3}, E_{D}$ and $E_{A}$ are the valence and the conduction tail slopes in $\mathrm{meV}, \sigma_{N}$ and $\sigma_{P}$ are the electron and hole cross sections of midgap states in $\mathrm{cm}^{2}, t_{C}$ and $t_{N}$ are the cross section of charged and neutral tail states in $\mathrm{cm}^{2}, N_{C}$ and $N_{V}$ are the effective DOS at the conduction band and at the valence band, respectively, in $\mathrm{cm}^{-3}, G_{\mathrm{DO}}$ and $G_{\mathrm{AO}}$ are the DOS at the mobility edges in $\mathrm{cm}^{-3} \mathrm{eV}^{-1}$, and $\mu_{N}$ and $\mu_{P}$ are the electron and hole mobilities in $\mathrm{cm}^{2} / \mathrm{V} \mathrm{s}$.

\begin{tabular}{lcccccccc}
\hline \hline Material & $E_{G}$ & $\mathrm{DB}$ & $E_{D}, E_{A}$ & $\sigma_{C}-\sigma_{N}$ & $t_{C}-t_{N}$ & $N_{C}, N_{V}$ & $\operatorname{DOS}\left(E_{C}, E_{V}\right)$ & $\mu_{N}, \mu_{P}$ \\
\hline (i) $a$-Si: $\mathrm{H}$ & 1.80 & $10^{16}$ & $51-30$ & $5 \times 10^{-15}$ & $9 \times 10^{-15}$ & $3 \times 10^{20}$ & $10^{21}$ & $9-0.5$ \\
& & & & $5 \times 10^{-16}$ & $2 \times 10^{-16}$ & & & \\
(i) $\mu c$-Si:H & 1.32 & $3.75 \times 10^{15}$ & $35-23$ & $6 \times 10^{-14}$ & $10^{-15}$ & $3 \times 10^{19}$ & $2 \times 10^{20}$ & $120-40$ \\
& & & & $6 \times 10^{-15}$ & $10^{-17}$ & & & \\
\hline \hline
\end{tabular}

some additional physics integrated in order to improve the characterization and design of single and multiple solar cells of amorphous, microcrystalline, and crystalline silicon. For instance we can mention the inclusion of amphoteric states, ${ }^{17}$ the Pool-Frenkel model, ${ }^{18}$ the defect pool model,,${ }^{19,20}$ direct tunneling at constant energy, ${ }^{21}$ recombination tunnelling, ${ }^{18}$ gap and doping continuous grading, light scattering at rough surfaces, light interference, ${ }^{22}$ etc. The density of states (DOS) in $a-\mathrm{Si}: \mathrm{H}$ and in $\mu c-\mathrm{Si}: \mathrm{H}$ is described by two exponential tails and by three Gaussian distributions recognized with the symbols $D^{-}, D^{o}$, and $D^{+}$. The correlation energy $U$ is assumed to be positive and equal to $0.2 \mathrm{eV}$. Tails host single donorlike and acceptorlike states and Gaussian host bivalent states. ${ }^{14,16,17,19,20}$ Since there is not yet a clear picture available for the distribution of energy offsets they were equally split between the conduction and the valence bands. ${ }^{19,20}$ Contact effects will not be discussed in this paper. Flat bands are assumed at both contacts. The electrical parameters adopted in our simulations resulted from extensive fittings of experimental characteristic curves $(J-V$ and SR) of $a-\mathrm{Si}: \mathrm{H} \quad p-i-n, \quad \mu c-\mathrm{Si}: \mathrm{H} \quad p-i-n, \quad$ and $\quad a-\mathrm{Si}: \mathrm{H} / a-\mathrm{Si}: \mathrm{H}$ $p-i-n / p-i-n$ solar cells. ${ }^{14,18-20,23}$ The most relevant electrical parameters used for the $a-\mathrm{Si}: \mathrm{H}$ and $\mu c-\mathrm{Si}: \mathrm{H}$ intrinsic layers are listed in Table I. They correspond to materials grown with the hot wire chemical vapor deposition (HWCVD) technique (see Sec. V for more details).

\section{DARK J-V CHARACTERISTIC CURVES OF SINGLE- JUNCTION SILICON P-I-N DEVICES}

\section{A. a-Si: $\mathrm{H}$ single-junction $p-i-n$ structures}

In previous publications we have discussed the conduction mechanisms controlling the dark current of $a-\mathrm{Si}: \mathrm{H}$ based $p-i-n$ devices $^{24,25}$ where we have $a-\mathrm{Si}: \mathrm{H}$ or $a-\mathrm{SiC}: \mathrm{H}$ $p$-doped layers and $a$-Si:H or $\mu c-\mathrm{Si}: \mathrm{H} n$-doped layers. We have identified five mechanisms acting in series: injection of holes and electrons at the front and back contacts, respectively, diffusion (below flat bands) or drift (above flat bands) of electrons and holes toward the bulk, and recombination of electron-hole (e-h) pairs inside the intrinsic layer. At very low forward voltages the dark current $J$ of $a-\mathrm{Si}: \mathrm{H} p-i-n$ is entirely controlled by recombination through midgap states in the intrinsic layer. ${ }^{24,25}$ The recombination rate reaches its maximum closer to the $p / i$ interface due to the lower mobility of holes. We recognize the voltage below which the dark current is entirely controlled by recombination $V_{R}$, where the subindex $R$ stands for recombination (see Fig. 1). The voltage $V_{R}$ defines also the onset of free carrier diffusion against the field along the intrinsic layer. For voltages exceeding $V_{R}$ but still inside the exponential region, the dark current becomes limited by the combination of diffusion and recombination., ${ }^{9,2426}$ The higher the voltage, the more the current becomes controlled by diffusion. This behavior has some similarities with the one observed in crystalline semiconductor $p$ - $n$ junctions. ${ }^{10,27}$ Hence we can define the second voltage region by the inequalities $V_{R}<V<V_{E}$, where $E$ stands for the end of the exponential region (see Fig. 1). At high forward voltages the dark $J-V$ curve rolls over and increases more slowly with the applied voltage. The device enters into the so-called electron SCLC regime. ${ }^{9,10}$ Although the current is controlled by electron diffusion over the virtual cathode the electron potential distribution around the virtual cathode is not only a function of the concentration of trapped electrons but also of the concentration of trapped holes. ${ }^{24,25}$ The SCLC regime is observed at voltages $V$ such as $V$ $>V_{\mathrm{SC}}\left(V_{\mathrm{SC}}\right.$ is the voltage associated with the initial formation of the virtual cathode), where $V_{\mathrm{SC}}>V_{E}$. The three voltages $V_{R}, V_{E}$, and $V_{\mathrm{SC}}$ (seen in Fig. 1) that are obtained from

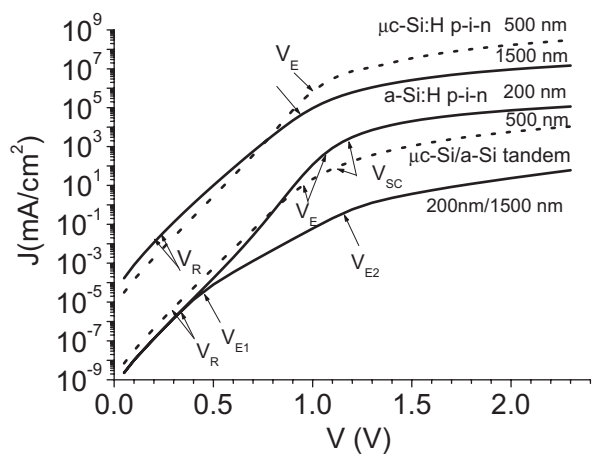

FIG. 1. Dark $J-V$ curves of $a$-Si:H $p-i-n$ single junctions, $\mu c-\mathrm{Si}: \mathrm{H} p-i-n$ single junctions, and of an $a-\mathrm{Si}: \mathrm{H} / \mu c-\mathrm{Si}: \mathrm{H}$ tandem structure. The intrinsic layer thicknesses are $200 \mathrm{~nm}$ (solid line) and $500 \mathrm{~nm}$ (dotted line) in the $a-\mathrm{Si}: \mathrm{H} p-i-n, 500 \mathrm{~nm}$ (dotted line) and $1500 \mathrm{~nm}$ (solid line) in the $\mu c-\mathrm{Si}: \mathrm{H}$ $p-i-n$, and 200 (top) and $1500 \mathrm{~nm}$ (bottom) in the subcells of the $a-\mathrm{Si}: \mathrm{H} / \mu c-\mathrm{Si}: \mathrm{H}$ tandem. In single junctions below the voltage $V_{R}$ the current is entirely controlled by recombination of e-h pairs. The voltage $V_{E}$ indicates the end of the exponential region and $V_{\mathrm{SC}}$ the beginning of the space charge regime. In the tandem structure the voltage range $0<V<V_{E 1}$ defines the first exponential region, the voltage range $V_{E 1}<V<V_{E 2}$ defines the second exponential region, and the voltage range $V>V_{E 2}$ the third region. Doped layers of $\mu c-\mathrm{Si}: \mathrm{H}$ are $20 \mathrm{~nm}$ thick. 
detailed simulations are complex functions of the intrinsic layer thickness, the density of midgap states, and the free carrier mobilities. The high forward dark $J-V$ of devices with front $p$-layer of $a-\mathrm{SiC}: \mathrm{H}$ (or $a-\mathrm{Si}: \mathrm{H}$ ) with activation energies higher than $0.35 \mathrm{eV}$ becomes controlled by hole injection instead of by SCLC. ${ }^{24}$

In this paper we analyze $a-\mathrm{Si}: \mathrm{H} p-i$ - $n$ devices prepared with the HWCVD technique. Both doped layers are made of $\mu c$-Si:H. The activation energies of the $p$-doped and $n$-doped layers were determined with temperature dependence of the dark conductivity as 0.072 and $0.045 \mathrm{eV}$, respectively. In these devices the corresponding condition to have a high forward current limited by SCLC and not by hole injection is that the sum of the $p-\mu c-\mathrm{Si}: \mathrm{H}$ activation energy and the valence band offset at the $p / i$ interface should not be much higher than $0.35 \mathrm{eV}^{20}$ This condition is usually fulfilled. ${ }^{28}$ High band-gap layers present at the $p / i$ interface could also hinder the maximum high forward current that can be achieved with these structures.

Other important difference between the structure discussed in this paper and the one previously studied is that the $a-\mathrm{Si}: \mathrm{H}$ intrinsic layers of the top subcell in tandems are much thinner than the intrinsic layers of regular $a-\mathrm{Si}: \mathrm{H}$ solar cells. However our simulations indicate that the transport mechanisms controlling the dark $J-V$ of these thin $a-\mathrm{Si}: \mathrm{H}$ $p-i-n$ devices are similar to the ones present in regular $a-\mathrm{Si}: \mathrm{H} p-i-n$ devices. Inside the exponential region the dark $J-V$ is controlled by recombination through gap states at very low voltages and by a combination of recombination and free carrier diffusion at higher voltages. Outside the exponential region the dependence of the dark $J$ - $V$ with respect to intrinsic layer thickness and voltage is different from the usual dependence reported in the literature for the SCLC regime for samples with intrinsic layers between 0.5 and $2 \mu \mathrm{m} .^{29}$ However we still see the formation of the virtual cathode for electrons limiting the electron injection into to the $i$-layer. Hence we conclude that the current is still controlled by SCLC. This issue will be discussed in more detail in a future publication.

The use of either $(n)-\mu c-\mathrm{Si}: \mathrm{H}$ or $(n)-a-\mathrm{Si}: \mathrm{H}$ layers does not introduce significant differences. For simplicity and due to the fact that there is not yet any evidence on the validity of the defect pool model in $\mu c-\mathrm{Si}: \mathrm{H}$ solar cells we will assume in this paper that the density of dangling bonds is just uniform inside the intrinsic $\mu c-\mathrm{Si}: \mathrm{H}$ and $a$-Si:H layers. We have checked that the conclusions of our study are valid when the DOS in (i)a-Si:H is evaluated with the defect pool model.

In Fig. 1 we show the computed dark $J$ - $V$ curve of $a$-Si:H based $p-i-n$ cells with 200 and $500 \mathrm{~nm}$ thick intrinsic regions. The first thickness corresponds to the top subcell intrinsic thickness. Both doped layers are of $\mu c-\mathrm{Si}: \mathrm{H}$. They were assumed to be $20 \mathrm{~nm}$ thick in order to electrostatically shield the bulk from external contacts.

\section{B. $\mu c-S i: H$ single-junction $p-i-n$ structures}

In $\mu c-\mathrm{Si}: \mathrm{H} p-i-n$ devices the same mechanisms control the dark $J-V$ curve at low forward biases: recombination and diffusion of free carriers. ${ }^{12-14,30}$ Similarly, the two voltages $V_{R}$ and $V_{E}$ can be defined in the exponential region. At high forward voltages there is some consensus in that the dark $J-V$ characteristic is controlled by series resistance when contacts do not hinder hole injection. ${ }^{12,13,30}$ The measurement of the dark $J-V$ at high forward voltages is particularly difficult in $\mu c$-Si:H $p-i-n$ devices due to the high current densities that could be achieved. Experimental setups are supplied with appropriate electronics to protect the equipment of excessive currents that might also damage the sample. We will show in Sec. IV that the high forward dark $J-V$ regime of $\mu c-\mathrm{Si}: \mathrm{H}$ $p-i-n$ devices does not play any role in defining the dark $J-V$ curve of micromorph solar cells in the voltage range under study.

The dark $J-V$ curves of $a-\mathrm{Si}: \mathrm{H}$ and $\mu c-\mathrm{Si}: \mathrm{H} p-i-n$ devices are compared in Fig. 1 for the same intrinsic layer thickness of $500 \mathrm{~nm}$. The other $\mu c-\mathrm{Si}: \mathrm{H} p-i-n$ dark $J-V$ corresponds to the intrinsic layer thickness of the bottom subcell of the tandem structure. The bottom cell of the tandem solar cell samples that we will discuss in this paper was also prepared with the HWCVD technique and deposited at hydrogen $(\mathrm{H})$ dilutions that lead to microcrystalline intrinsic layers which do not depart significantly from a homogeneous structure as revealed from the inspection of cross sectional transmission electron microscopy micrographs. ${ }^{31}$ Intrinsic layers deposited with plasma-enhanced chemical-vapor deposition can also be quite uniform when the $\mathrm{H}$ concentration is tailored with H-profiling techniques. Grain sizes are uniform and so are the electrical properties. ${ }^{32}$ For this particular paper we try to keep our modeling as simple as possible in order to reach general conclusions about the dark $J$ - $V$ curves of tandem cells that are already complex devices. In Sec. V we show that we could fit the dark $J-V$ of a single-junction $\mu c-\mathrm{Si}: \mathrm{H} p-i-n$ device with a $1400 \mathrm{~nm}$ thick intrinsic layer prepared under conditions which are similar to the bottom subcell of the micromorph solar cell by assuming uniform electrical properties (see Table I). We verified that our conclusions do not change when the electrical properties in the intrinsic layer are assumed to be nonuniform. We could also say that the parameters of Table I correspond to effective average electrical properties.

\section{DARK J-V CHARACTERISTIC CURVES OF $N-I-P$ TANDEM RECOMBINATION JUNCTIONS}

Microcrystalline silicon $(\mu c-\mathrm{Si})$ is a very interesting material for TRJs of multijunction solar cells. Its low mobility gap and optical absorption favor recombination and minimize optical losses, respectively. Under illumination electrons and holes photogenerated in the top and bottom subcells are injected into the $n-i-p$ TRJ where they recombine. This process recognized as "good" recombination sustains the photocurrent present in the tandem solar cell. ${ }^{16,18}$ One of the tasks of an efficient TRJ is to provide enough good recombination to allow for the full recombination of the free carriers photogenerated in both the subcells. It is well known that the classical Shockley-Read-Hall (SRH) formalism is not able to reproduce the experimental tandem solar cell open circuit voltage (Voc) and fill factor (FF). ${ }^{18,33,34}$ Hou et 
$a l .{ }^{33}$ and Bae and Fonash ${ }^{35}$ graded the mobility gap but there was no experimental evidence supporting this physics. Willemen $^{34}$ was the first in successfully fitting the light $J-V$ of tandem solar cells using computer modeling with some experimental support. He incorporated the trap assisted tunneling (TAT) recombination formalism developed by Hurkx et $a l^{36}$ to model dark $J-V$ of highly doped step junctions. He found that Voc increased but the predicted $J-V$ curves were still quite off from the measured ones. ${ }^{34}$ A gradient in the quasi-Fermi levels was needed to move electrons and holes from the position where they were generated to where the good recombination was taking place. Then Willemen invoked the exponential dependence observed in the drift mobility $\mu_{\text {eff }}$ with respect to electric field $F$, i.e., $\mu_{\text {eff }}$ $=\mu_{o} \exp \left(F / F_{o}\right)$, where $\mu_{o}$ is the regular free carrier mobility and $F_{o}(\mathrm{~V} / \mathrm{cm})$ is a parameter that determines the strength of this field dependence. ${ }^{37}$ Combining TAT with field dependent mobilities he could reproduce the measured $J$ - $V$ characteristics of tandem solar cells. ${ }^{34}$

In a previous publication we studied the dark $J-V$ curves of single $n-i-p \mu c$-Si TRJ structures and the light $J-V$ of $a$-Si tandem cells with different models available in the literature: ${ }^{18}$ TAT, the Pool-Frenkel effect (PFE) ${ }^{38}$ field dependent cross sections only (FDCSs) ${ }^{37}$ and field dependent attempt to escape frequencies $\nu$ (FDAEF) following the work of Gu et al. ${ }^{39}$ i.e., $\nu_{\text {eff }}=\nu_{o} \exp \left(F / F_{o}\right)$. This last approach is equivalent to simultaneously assume field dependent free carrier mobilities and field dependent cross sections (or effective density of states $N_{c}$ and $N_{v}$ ). Field dependent cross section formalisms (PFE, FDCS, and FDAEF) significantly enhance the thermal generation of e-h pairs predicted by the SRH formalism but these magnifications are performed on single grid points. Field dependent mobilities were still needed to reproduce the shape of the experimental dark $J-V$ characteristics of single TRJ that show an Ohmic behavior at low voltages. ${ }^{40}$ Our result was similar to the findings of Willemen. ${ }^{34}$ The model proposed by Gu and Schiff ${ }^{39}$ was the most appropriate to reproduce the behavior of the experimental dark $J-V$ of TRJ. The ideas used to match the light $J-V$ of tandem cells and dark $J-V$ of TRJ will be adopted used in this paper to model and reproduce the experimental dark $J-V$ of tandem cells.

In tandem solar cells under AM1.5 illumination the injected photogenerated carriers that cannot recombine become trapped at gap states. The piling up of trapped charges on both sides of the $n-i-p$ structure forward biases the TRJ under bias light. Under dark conditions, in contrast, the TRJ $n-i-p$ structure becomes reverse biased by the external forward voltage applied to the tandem structure. Free holes and electrons coming from the top and from the bottom subcells, respectively, are injected into the TRJ. In order to close the current path and sustain the dark current the TRJ must thermally generate enough e-h pairs to inject electrons and holes into the top and into the bottom subcells, respectively. Electrons and holes injected from the bottom and top subcells, respectively, into the TRJ are not enough to sustain the dark current of the tandem structure. These mechanisms are sketched in Fig. 2 for a tandem cell subjected to a moderate forward bias.

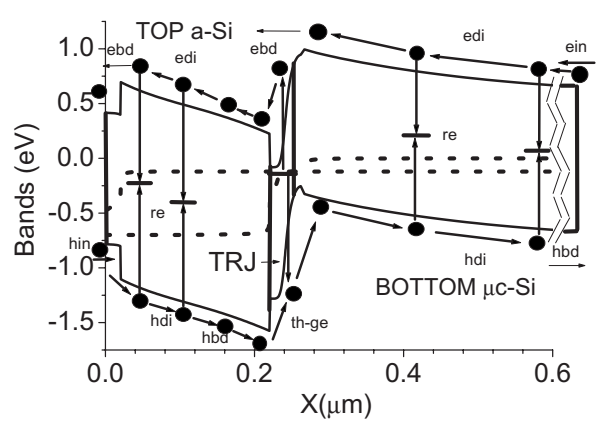

FIG. 2. Band diagram showing the transport mechanisms present in an $a-\mathrm{Si}: \mathrm{H} / \mu c-\mathrm{Si}: \mathrm{H}$ tandem under dark conditions. Meaning of symbols are as follows: (ebd) electron back diffusion, (hbd) hole back diffusion, (edi) electron diffusion, (hdi) hole diffusion, (re) recombination of e-h pairs, and (th-ge) thermal generation of e-h pairs. The applied voltage is of $0.7 \mathrm{~V}$.

In reverse biased single $p-i-n$ or $n-i-p \quad a-\mathrm{Si}: \mathrm{H}$ or $\mu c-\mathrm{Si}: \mathrm{H}$ devices the dark current is supplied by $\mathrm{e}-\mathrm{h}$ pairs thermally generated by gap states. ${ }^{24,41}$ At low reverse voltages the generation of e-h is concentrated in a small region of the intrinsic layer situated closer to the $p / i$ than to the $i / n$ interface. When the reverse voltage is increased the generation of e-h spreads toward the doped layers. Thermally generated electrons and holes exit the device pushed by the electric field. The physics of $\mu c-\mathrm{Si}: \mathrm{H} n-i-p$ TRJ structures has particular aspects that are not found in regular $\mu c-\mathrm{Si}: \mathrm{H}$ devices due to its small intrinsic layer thickness $(2-6 \mathrm{~nm})$. The model of $\mathrm{Gu}$ and Schiff ${ }^{39}$ predicts a significant dependence of the enhanced emission rates with respect to the electric field $F$ when $F$ becomes comparable to the parameter $F_{o}$. The electric field profile is independent of the value adopted for $F_{o}$. The electric field in tandem TRJ can reach values near $10^{6} \mathrm{~V} / \mathrm{cm}$. The e-h pairs are mostly generated inside the intrinsic layer and in the first nanometer of the $p$-layer by the $i / p$ interface. This result was also obtained for the recombination of e-h pairs taking place in the TRJ of tandem solar cells under illumination conditions. ${ }^{16,18}$

Figure 3 shows the dependence of the dark $J-V$ with respect to the parameter $F_{o}$ predicted by D-AMPS for isolated $n-i-p \mu c-S i: H$ TRJ. The basic parameters used in these simulations are listed in Table II. The thermal generation rate and the total dark current are strongly dependent on the parameter $F_{o}$ (Ref. 39) and on the mobility gap. They are also

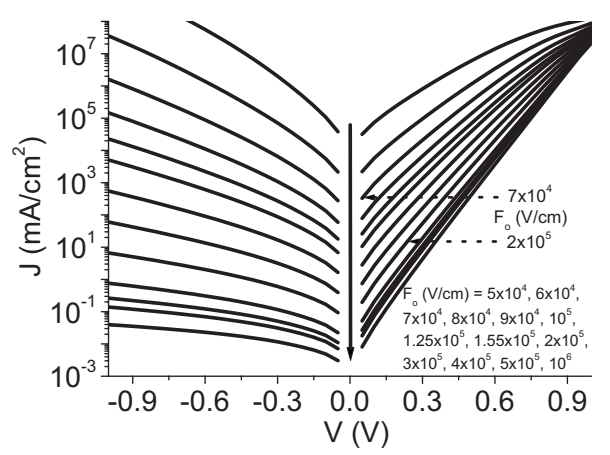

FIG. 3. Dependence of the dark $J$ - $V$ characteristics of the $\mu c$-Si $n$ - $i$ - $p$ TRJ with respect to the parameter $F_{o}$. Doped layers are $20 \mathrm{~nm}$ thick and the $i$-layer thickness is assumed to be $5 \mathrm{~nm}$ thick. The arrow with the solid line is a guide to the eye that indicates the direction of increasing values of $F_{o}$. Arrows with dotted lines correspond to values of $F_{o}$ discussed in the text. 
TABLE II. List of the electrical parameters of the TRJ structure. The meaning of the symbols are as the ones present in Table I. $W$ is the layer thickness in nanometers.

\begin{tabular}{|c|c|c|c|c|c|c|c|c|c|}
\hline Material & $E_{G}$ & DB & $E_{D}, E_{A}$ & $\sigma_{C}-\sigma_{N}$ & $t_{C}-t_{N}$ & $N_{C}, N_{V}$ & $\operatorname{DOS}\left(E_{C}, E_{V}\right)$ & $\mu_{N}, \mu_{P}$ & $W$ \\
\hline (i) $\mu c-\mathrm{Si}: \mathrm{H}$ & 1.2 & $2.5 \times 10^{18}$ & $30-20$ & $\begin{array}{l}5 \times 10^{-14} \\
5 \times 10^{-15}\end{array}$ & $\begin{array}{l}10^{-14} \\
10^{-16}\end{array}$ & $3 \times 10^{19}$ & $2 \times 10^{20}$ & $40-4$ & 2 \\
\hline $\begin{array}{l}\text { (Doped) } \\
\mu c-\mathrm{Si}: \mathrm{H}\end{array}$ & 1.2 & $5 \times 10^{18}$ & $30-20$ & $\begin{array}{l}5 \times 10^{-14} \\
5 \times 10^{-15}\end{array}$ & $\begin{array}{l}10^{-14} \\
10^{-16}\end{array}$ & $3 \times 10^{19}$ & $2 \times 10^{20}$ & $40-4$ & 20 \\
\hline
\end{tabular}

sensitive but in less extent to the dangling bond (DB) density and thickness of the intrinsic layer. An efficient tandem solar cell should not have a significant voltage drop in the TRJ structure under illumination conditions. The short circuit current of a tandem cell is between $8-11 \mathrm{~mA} / \mathrm{cm}^{2}$. The inspection of Fig. 3 indicates that the parameter $F_{o}$ should not be higher than $2 \times 10^{5} \mathrm{~V} / \mathrm{cm}$. On the other hand, extreme values of $F_{o}$ below $7 \times 10^{4} \mathrm{~V} / \mathrm{cm}$ would give rise to unreasonable high dark current levels that are not experimentally observed. ${ }^{37,40}$ The field enhancement of the thermal generation rate of e-h pairs is lost for very thick intrinsic layers and the current level does not constantly increase for thinner and thinner intrinsic layers because doped layers take finally full control of the generation of e-h pairs.

\section{DARK J-V CHARACTERISTIC CURVES OF THE MICROMORPH TANDEM SOLAR CELL}

The list of possible transport mechanisms that under dark conditions defines the shape of $J-V$ characteristic of a micromorph silicon tandem cell results from combining the mechanisms already described in Secs. II and III. Going from the front contact toward the back contact we can list the following nine mechanisms:

(1) Hole injection through the front contact.

(2a) Hole diffusion against the field direction in the $p$-layer and $i$-layer of the top cell.

(2b) Hole diffusion along the field direction over the virtual anode in the top cell.

(3) e-h recombination in the $i$-layer of the top cell.

(4a) Electron diffusion against the field direction in the $n$-layer and $i$-layer of the top cell.

(4b) Electron diffusion along the field direction over the virtual cathode of the top cell.

(5) Thermal generation of e-h pairs at the TRJ.

(6a) Hole diffusion against the direction of the field in the bottom cell.

(6b) Hole diffusion in the field direction limited by series resistance.

(7) e-h recombination in the $i$-layer of the bottom cell.

(8a) Electron diffusion against the direction of the field in the bottom cell.

(8b) Electron diffusion in the field direction limited by series resistance.

(9) Electron injection through the back contact.
The subindex $a$ corresponds to voltages below the flat band condition and the subindex $b$ corresponds to voltages above the flat band condition. As the tandem solar cell is composed of three single junctions connected in series the distribution of the applied voltage must also be investigated. The device under analysis has the following structure: $p$ - $\mu c-\mathrm{Si} / i-a-\mathrm{Si} / n-\mu c \mathrm{Si} / i-\mu c-\mathrm{Si} / p-\mu c-\mathrm{Si} / i-\mu c-\mathrm{Si} / n-\mu c-\mathrm{Si}$.

The intrinsic layer thicknesses of the top and the bottom cells were 200 and $1500 \mathrm{~nm}$, respectively, in agreement with the values of the sample that will be discussed in Sec. V. The voltage drop predicted by our computer code over the three single junctions of this particular device is showed in Fig. 4. Our results are presented for different values of $F_{o}(\mathrm{~V} / \mathrm{cm})$. A higher fraction of the applied voltage drops on the top cell due to the lower conductivity of $a$-Si with respect to $\mu c$-Si. It is important to note that a non-negligible voltage drop could take place on the TRJ especially for higher values of $F_{o}$ that correspond to a nonoptimized TRJ. The forward biased tandem cell has two forward biased single $p-i$ - $n$ junctions and one reverse biased $n-i-p$ junction (TRJ). The TRJ structure must provide to the top cell enough electrons to sustain the dark current in conjunction with the holes injected through the front contact. These electrons are thermally generated inside the TRJ and must diffuse through the $n$-layer of the top subcell into the intrinsic layer. The TRJ structure must also provide to the bottom cell enough holes to sustain the dark current in conjunction with the electrons injected through the back contact. These holes are also thermally generated in the TRJ and must diffuse through the $p$-layer of the bottom subcell into the intrinsic layer (Fig. 2). For higher values of $F_{o}$ the TRJ generates less e-h pairs due to field dependence of the cross sections present in Gu's model. ${ }^{39}$

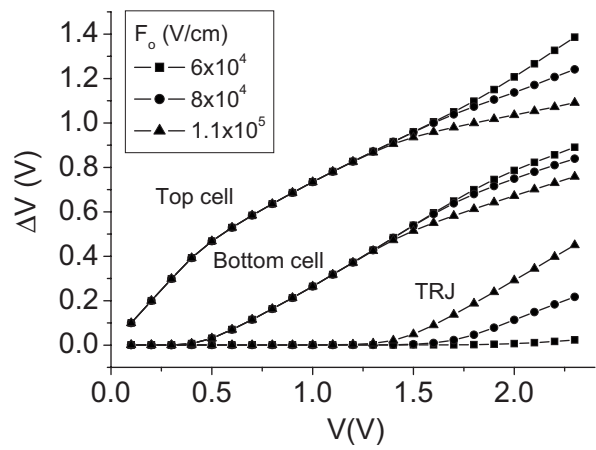

FIG. 4. Voltage drop $\Delta V$ on the top subcell, on the bottom subcell, and on the TRJ of the $a-\mathrm{Si}: \mathrm{H} / \mu c-\mathrm{Si}: \mathrm{H}$ tandem cell with respect to the total applied voltage and for different values of the parameter $F_{o}$. The intrinsic layer thicknesses are 200 and $1500 \mathrm{~nm}$. The voltage drop on the TRJ is plotted in absolute value. 

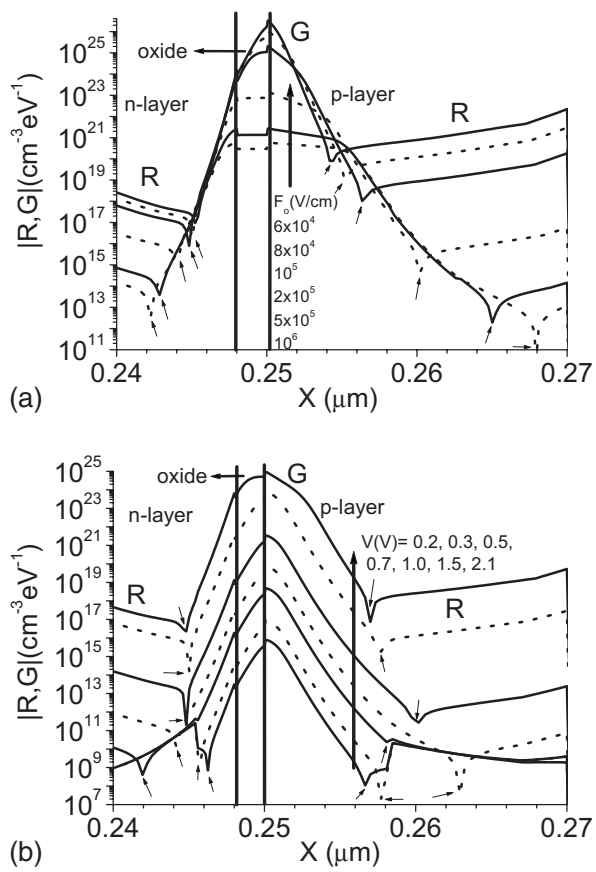

FIG. 5. (a) Dependence of the recombination rate and of the generation rate profiles of e-h pairs inside the $\mu c$-Si $n-i-p$ TRJ with respect to the parameter $F_{o}(\mathrm{~V} / \mathrm{cm})$. The thermal generation rate is showed in absolute value. The external voltage applied to the tandem junction was fixed to $2.1 \mathrm{~V}$. The arrow is a guide to eye that indicates the direction of increasing values of $F_{o}$. (b) Dependence of the recombination rate and of the generation rate profiles of e-h pairs inside the $\mu c-\mathrm{Si} n-i-p$ TRJ with respect to the external voltage $V$ applied to the tandem solar cell. The thermal generation rate is showed in absolute value. The parameter $F_{o}(\mathrm{~V} / \mathrm{cm})$ is fixed to $1.1 \times 10^{5} \mathrm{~V} / \mathrm{cm}$. The arrow is a guide to the eye that indicates the direction of increasing values of $V$. Thin arrows enclose the region where thermal generation overcomes recombination.

Hence the higher the parameter $F_{o}$, the more reversed biased the TRJ device will become in order to thermally generate more e-h pairs in an attempt to sustain the total dark current. In other words a nonefficient thermal generation of e-h pairs in the TRJ would lead to a significant drop in the external applied voltage in the TRJ and to lower voltage drops in the subcells. A similar statement can be made for higher band gaps, less defective and thicker TRJs. Figure 5(a) illustrates the dependence of the generation rate inside the TRJ of a micromorph solar cell with respect to $F_{o}$ for a given value of the external applied voltage. Actually D-AMPS output gives the recombination rate $R$ of e-h pairs. As the thermal generation in output is given as a negative $R$ we show in this figure the absolute value of $R$. The region where generation overcomes recombination is indicated by thin arrows. Figure 5(b) shows the dependence of the thermal generation rate with respect of the external applied voltage for a given value of $F_{o}$. The thermal generation of e-h pairs increases for higher forward voltages making possible the flow of higher dark currents at higher forward voltages [Fig. 5(b) is presented in the same format as Fig. 5(a)].

Typical intrinsic layer thicknesses of micromorph cells are in the range of 150-200 $\mathrm{nm}$ for the top subcell and in the range of 1000-2000 nm for the bottom subcell. Figure 1 indicates that in $(i)-\mu c-\mathrm{Si} p-i-n$ the voltage $V_{E}$ where the regime of the current limited by series resistance begins is around 0.9 V. Inspecting Fig. 4 we can see that in the micro- morph tandem cell the bottom subcell would never reach $V_{E}$ within the voltage range under study. Hence we can exclude mechanisms (6b) and (8b) from our list. The top cell might not reach the SCLC when the TRJ structure takes a significant fraction of the applied voltage (see Figs. 1 and 4). Hence mechanisms (2b) and (4b) might not be active either in tandem cells that rely on nonoptimized TRJ.

Examining Fig. 1 we can clearly distinguish three different regions in the dark $J-V$ characteristics of the micromorph tandem cells. They are defined by the voltage intervals 0 $<V<V_{E 1}$ (first), $V_{E 1}<V<V_{E 2}$ (second), and $V>V_{E 2}$ (third). In the device under study these voltages are $V_{E 1}$ $\sim 0.5 \mathrm{~V}$ and $V_{E 2} \sim 1.2 \mathrm{~V}$. In the first two regions the current increases exponentially with respect to the applied voltage but with different diode factors, and in the third region the current increases more slowly with the applied voltage (see Fig. 1). The diode factors in the exponential regions should not have necessarily a direct correspondence with the diode factors of the exponential regions of the single $a-\mathrm{Si}: \mathrm{H}$ and $\mu c$-Si $p-i-n$ devices because the applied voltage is distributed over both subcells. However in the first region and at very low forward voltages such as $0<V<V_{\mathrm{TD}}$ ( $\mathrm{T}$ stands for top cell) $\left(V_{\mathrm{TD}} \sim 0.38 \mathrm{~V}\right)$ the diode factor of the tandem cell is very similar to the diode factor of the top subcell due to the fact that most of the applied voltage drops on the top cell. The current becomes limited by the top subcell because the dark current of the tandem cell is below the minimum current that can be extracted from the single bottom subcell (see Fig. 1). Interestingly in the second exponential region it is easy to show by using simple equivalent circuit analysis that the diode factor of the tandem cell is given by the sum of the diode factor of the two single $p-i-n$ cells.

In order to establish which transport mechanisms really define the shape of the dark $J$ - $V$ curve of the micromorph solar cell detailed modeling and sensitivity studies were conducted. For the lowest voltages of the first exponential region, i.e., for $V$ such as $0<V<V_{\mathrm{TD}}$, the dark current is practically controlled by recombination of e-h pairs through gap states of the intrinsic $a-\mathrm{Si}: \mathrm{H}$ layer. At the highest voltages but still inside the first exponential region diffusion of free carriers at the top cell plays also a role. The voltage $V_{\mathrm{TD}}$ corresponds to the onset of diffusion of free carriers in the top cell. There is a minor contribution coming from recombination of e-h pairs through gap states of the intrinsic $\mu c$-Si. The onset of recombination through gap states of the bottom cell is a bit below $V_{\mathrm{E} 1}$ and it would be recognized with the symbol $V_{\mathrm{BR}}(\sim 0.45 \mathrm{~V})$. In the second exponential region the current is controlled by the combination of three mechanisms: recombination through gap states in the $(i)-a-\mathrm{Si}: \mathrm{H}$ layer (top cell), recombination through gap states in the (i)- $\mu c$-Si layer (bottom cell), and diffusion of free carriers in the top cell. Finally in the third region the current is controlled by three mechanisms: thermal generation of e-h pairs in the TRJ, recombination of free carriers in the bottom cell, and a minor contribution of free carrier diffusion along the same cell. Only when low values of $F_{o}$ are adopted in the TRJ structure the onset of the SCLC regime in the top cell could be reached within the voltage range under study (see 


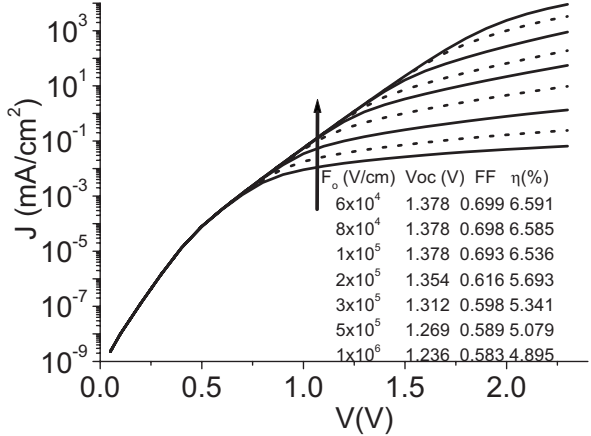

FIG. 6. Dependence of the $a-\mathrm{Si}: \mathrm{H} / \mu c-\mathrm{Si}: \mathrm{H}$ tandem dark $J-V$ characteristics with respect to the parameter $F_{o}$. A brief table with the solar cell performance achieved for different values of $F_{o}$ is included for comparison The arrow is a guide to the eye that indicates the direction of increasing values of $F_{o}$.

Fig. 1). In this case the voltage range $V>V_{\mathrm{TSC}}(\sim 2 \mathrm{~V})$ could be defined (where TSC stands for top cell SCLC).

The dark $J$ - $V$ is insensitive to cross sections and mobilities of doped layers with one exception: the ones of the $p$-layer of the bottom cell. Inside the TRJ the thermal generation rate is directly proportional to cross sections. Higher mobilities help in the flow of free carriers out of the TRJ. However we must stress that the total current is sensitive to changes in cross sections and mobilities only in the intrinsic layer and in the first nanometer of the $p$-layer near the $i / p$ interface where thermal generation of e-h reaches significant values. There is no sensitivity of the total current with respect to these parameters in regions of the TRJ where recombination takes over thermal generation or where thermal generation is comparatively much lower than the peak value like, for instance, in the $n$-layer of the top subcell (see Fig. 5). Regions of the TRJ where the field and the generation rate are high are responsible for the sensitivity of the total dark current with respect to cross sections and mobilities of the TRJ $p$-layer and $i$-layer. This increase in the current is connected to a more efficient generation of e-h pairs and not to the transport of free carriers across the bottom cell. To clarify even further this concept we show in Fig. 6 the dependence of the $J-V$ curve of the micromorph cell with respect to the parameter $F_{O}$ that significantly impact in the amount of thermally generated e-h pairs inside the TRJ [see
Fig. 5(a)]. Figure 6 shows, as expected, that the dark $J$ - $V$ for high forward voltages is very sensitive to the rate of thermally generated e-h pairs occurring inside the TRJ. This is a quite distinctive and unique feature of the dark $J-V$ curves of tandem solar cells in comparison to their counterparts of single structures. In Fig. 6 we also include a short list of the solar cell performances for different values of $F_{O}$. We can see a strong correlation between higher dark currents at high forward voltages and a better efficiency of the solar cell. There is a saturation of the solar cell efficiency for lower values of $F_{O}$ such as $F_{O} \leq 8 \times 10^{4} \mathrm{~V} / \mathrm{cm}$; this saturation is not observed in the dark $J-V$ because the current levels reached at high forward voltages are much higher than under AM1.5 illumination and short circuit conditions. The device under analysis has not been optimized as solar cell. No back reflector has been included and the intrinsic layer thicknesses were not adjusted to achieve the maximum efficiency. Table III lists the solar cell performance parameters for this device and for a micromorph tandem solar cell that has been optimized.

As we already mentioned the generation of e-h pairs takes place mainly in the intrinsic layer of the TRJ and in the first nanometer of the $p$-layer (Fig. 2), i.e., exactly the same regions where the good recombination reaches its maximum values when the same tandem solar cell is subject to AM1.5 illumination. ${ }^{16,18}$ The position of the good recombination peak is dictated by the asymmetry of the $(n) \mu c-\mathrm{Si}$ and (p) $\mu c$-Si activation energies. The high recombination rates are found inside the doped region with the highest activation energy and in the TRJ intrinsic layer. When the doped $\mu c-\mathrm{Si}$ layer activation energies are exchanged the position of the good recombination rate peak under sun illumination conditions shifts from the region near the $i / p$ interface to the region near the $n / i$ interface. The same result is obtained under dark conditions for the thermal generation rate peak. The peaks of the good recombination under illumination and of the thermal generation rate under dark conditions are approximately located at the position where the Fermi level crosses midgap at thermodynamic equilibrium.

More defects, lower mobility band-gaps $E_{G}(\mathrm{eV})$, or lower values for $F_{o}(\mathrm{~V} / \mathrm{cm})$ in the intrinsic layer of the TRJ junction enhance the good recombination under illumination conditions and the thermal generation rate of e-h under dark

TABLE III. Open circuit voltage, fill factor, short circuit current and efficiency of the micromorph solar cell for different values of the parameter $F_{O}$. The upper half of the table corresponds to a solar cell with optimized intrinsic layer thicknesses and back reflector. The lower half of the table corresponds to the nonoptimized solar cell studied in this paper.

\begin{tabular}{lccccccccc}
\hline \hline$F_{o}(\mathrm{~V} / \mathrm{cm})$ & $6 \times 10^{4}$ & $8 \times 10^{4}$ & $1 \times 10^{5}$ & $1.25 \times 10^{5}$ & $1.5 \times 10^{5}$ & $2 \times 10^{5}$ & $3 \times 10^{5}$ & $5 \times 10^{5}$ & $1 \times 10^{6}$ \\
\hline Voc $(\mathrm{V})$ & 1.430 & 1.430 & 1.430 & 1.427 & 1.423 & 1.401 & 1.354 & 1.308 & 1.275 \\
$\mathrm{FF}$ & 0.714 & 0.713 & 0.707 & 0.679 & 0.656 & 0.626 & 0.608 & 0.599 & 0.593 \\
$J S c\left(\mathrm{~mA} / \mathrm{cm}^{2}\right)$ & 10.05 & 10.05 & 10.05 & 10.04 & 10.04 & 10.03 & 10.02 & 10.01 & 9.999 \\
$\eta(\%)$ & 10.27 & 10.26 & 10.16 & 9.745 & 9.367 & 8.792 & 8.243 & 7.844 & 7.563 \\
& & & & & & & & & \\
\hline$F_{o}(\mathrm{~V} / \mathrm{cm})$ & $6 \times 10^{4}$ & $8 \times 10^{4}$ & $1 \times 10^{5}$ & $1.25 \times 10^{5}$ & $1.5 \times 10^{5}$ & $2 \times 10^{5}$ & $3 \times 10^{5}$ & $5 \times 10^{5}$ & $1 \times 10^{6}$ \\
Voc $(\mathrm{V})$ & 1.378 & 1.378 & 1.378 & 1.376 & 1.372 & 1.354 & 1.312 & 1.269 & 1.236 \\
FF & 0.698 & 0.698 & 0.693 & 0.669 & 0.646 & 0.616 & 0.598 & 0.589 & 0.583 \\
$J S c\left(\mathrm{~mA} / \mathrm{cm}^{2}\right)$ & 6.845 & 6.847 & 6.846 & 6.839 & 6.833 & 6.821 & 6.808 & 6.797 & 6.789 \\
$\eta(\%)$ & 6.591 & 6.585 & 6.537 & 6.295 & 6.057 & 5.693 & 5.341 & 5.079 & 4.895 \\
\hline \hline
\end{tabular}



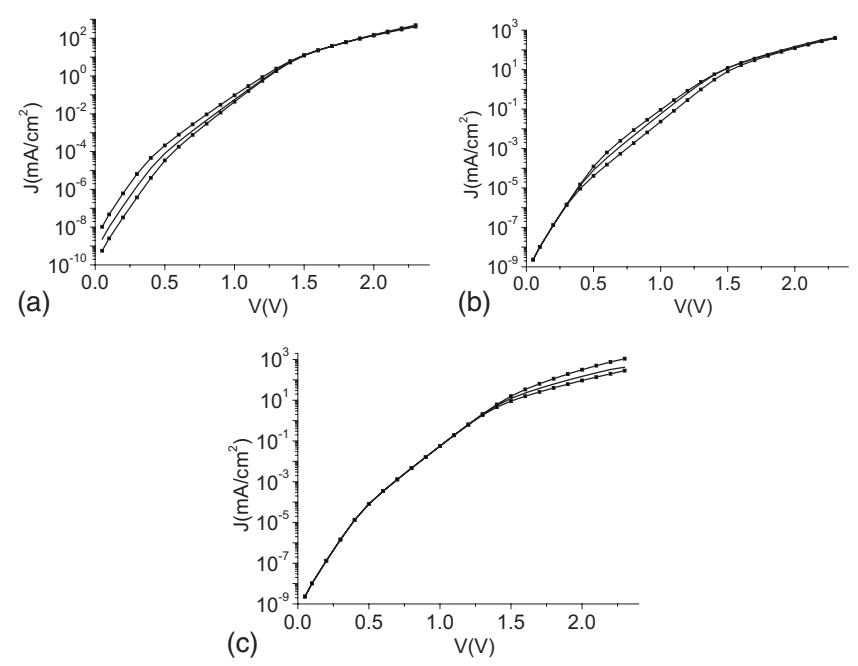

FIG. 7. (a) Dependence of the $a-\mathrm{Si}: \mathrm{H} / \mu c-\mathrm{Si}: \mathrm{H}$ tandem dark $J-V$ characteristics with respect to the DB density present in the top intrinsic layer. (b) Dependence of the $a-\mathrm{Si}: \mathrm{H} / \mu c-\mathrm{Si}: \mathrm{H} o$ tandem dark $J-V$ characteristics with respect to the DB density present in the bottom intrinsic layer. (c) Dependence of the $a-\mathrm{Si}: \mathrm{H} / \mu c-\mathrm{Si}: \mathrm{H}$ tandem dark $J-V$ characteristics with respect to the DB density present in the region of the TRJ where thermal generation is significant. The curves with square symbols correspond to density of DB that has been increased and decreased four times with respect to the value used in the dark $J-V$ represented with a solid line. The curves with square symbols correspond to density of DB that has been increased and decreased four times with respect to the value used in the dark $J-V$ represented with a solid line.

conditions. Under dark conditions more holes and electrons would be injected by the TRJ into the top and bottom cells, respectively, raising the current flowing through the tandem cell. These results indicate that the dark current of tandem cells at high forward voltages can be used to obtain some information on the actual value of the parameter $F_{o}(\mathrm{~V} / \mathrm{cm})$ when the density of DB, the mobility gaps $E_{G}$, and the layer thicknesses of the TRJ are known. The dark $J-V$ characteristic of tandem cells at high forward voltages can be also used as diagnosis tool to quickly inspect the efficiency of TRJ in recombining electron and holes under illumination. The higher the dark current achieved at high forward voltages, the more efficient the TRJ would be in recombining electrons and holes under illumination.

To conclude this section we show in Fig. 7 the dependence of the dark $J-V$ characteristic of the micromorph solar cell with respect to variations in the density of DB that were performed on different regions of the tandem solar cell. The DB densities have been increased and decreased by a factor of 4 with respect to the value used in Fig. 1. Figure 7(a) points out the high sensitivity of the dark current with respect to the DB density present in the intrinsic layer of the top subcell in the first exponential region $\left(V<V_{E 1}\right)$, where the dark current is mainly controlled by recombination through gap states of $a-\mathrm{Si}: \mathrm{H}$. This sensitivity extends to the second exponential region $\left(V_{E 1}<V<V_{E 2}\right)$ but it becomes less intense than that observed in the first exponential region. We have to keep in mind that in this region the current is controlled by three different mechanisms, recombination through gap states of $a-\mathrm{Si}: \mathrm{H}$ being only one of them. Figure 7(b) shows that the highest sensitivity of the dark current with respect to the density of $\mathrm{DB}$ present in the intrinsic layer of the bottom subcell is found in the second exponential region $\left(V_{E 1}<V<V_{E 2}\right)$, where recombination through gap states of $\mu c-\mathrm{Si}$ is another of the three mechanisms that control the total dark current. Some residual sensitivity of the dark current is observed in neighboring voltage regions ( $V$ $<V_{E 1}$ and $V>V_{E 2}$ ) outside of the second exponential region. Finally Fig. 7(c) shows the sensitivity of the dark $J$ - $V$ curve at the high voltages $\left(V>V_{E 2}\right)$ with respect to the density of DB present in the regions of the TRJ, where thermal generation of e-h pairs is significant. A lower sensitivity of the dark current to this mechanism is also observed only at the highest voltages of the second exponential region $\left(V_{E 1}<V\right.$ $\left.<V_{E 2}\right)$. The dark $J-V$ characteristic shows a minor sensitivity to the intrinsic layer thickness of the top cell in the voltage range $V<V_{E 1}$ and to the intrinsic layer thickness of the bottom cell in the voltage range $V_{E 1}<V<V_{E 2}$.

\section{COMPARISON WITH EXPERIMENTAL DARK $J-V$ CHARACTERISTICS}

In this section we fit the experimental dark $J$ - $V$ characteristic of a micromorph Si tandem solar cell grown with the HWCVD technique in the ultrahigh vacuum multichamber system PASTA at Utrecht University (UU). The solar cell was deposited on stainless steel (SS) in the substrate $n-i-p / n-i-p$ configuration. The device structure is as follows: $\quad \mathrm{Au} / \mathrm{ITO} / p-\mu c-\mathrm{Si} / \mathrm{TBL} / i-a-\mathrm{Si} / n-a-\mathrm{Si} / n-\mu c-\mathrm{Si} /$ $\mathrm{AB} / p-\mu c-\mathrm{Si} / \mathrm{TBL} / i-\mu c-\mathrm{Si} / n-a-\mathrm{Si} / \mathrm{ZnO} / \mathrm{Ag} / \mathrm{SS}$. TBL stand for thin buffer layer and $A B$ for the air break that leads to the formation of an oxide layer. We showed in a previous publication that similar performances can be achieved by either using a thin defective intrinsic $\mu c$-Si layer or an oxide layer between the doped $\mu c$-Si regions of the TRJ structure. We also showed that the oxide layer can be modeled as a defective intrinsic $\mu c$-Si layer. ${ }^{16}$ A $200 \mathrm{~nm}$ thick $i$ - $a$-Si:H layer was deposited at about $10 \AA$ /s using pure silane as source gas. The $i-\mu c-\mathrm{Si}$ layer was grown $\sim 1500 \mathrm{~nm}$ thick with a hydrogen dilution $\mathrm{H}_{2} /\left(\mathrm{SiH}_{4}+\mathrm{H}_{2}\right)$ of $0.952 .{ }^{42}$ All doped layers were made of $\mu c$-Si. The defect density of the intrinsic microcrystalline silicon layer obtained in this fitting is comparable to the defect density measured by Fourier transform photoconductivity spectroscopy in a single junction bottom cell. $^{43}$ Tail slopes and the global density of defects of the layers on glass were measured with the constant photocurrent method. The intrinsic layer thicknesses were estimated from the time of deposition. The values used in our simulations are within the experimental uncertainty. The experimental dark $J-V$ curves of the HWCVD made thin film silicon solar cells were measured at the UU laboratory. ${ }^{44}$

Our strategy was to fit first the dark $J-V$ characteristics of single $a-\mathrm{Si}: \mathrm{H}$ and $\mu c-\mathrm{Si} p-i$ - $n$ structures fabricated in the same reactor and under similar deposition conditions. Figure 8 shows the experimental dark $J$ - $V$ curves of these devices and our matches. They are cells with intrinsic layer thicknesses of $500 \mathrm{~nm}$ for the $i-a-\mathrm{Si}: \mathrm{H}$ layer and $1400 \mathrm{~nm}$ for the $i$ - $\mu c$-Si layer, respectively. Using the electrical parameters resulting from these fittings as baseline values for the tandem solar cell structure we found in our first attempt (making no changes at all) a good match of the dark $J-V$ curve in most of 


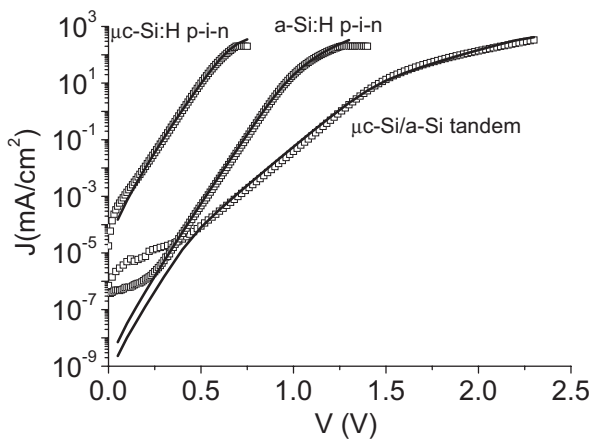

FIG. 8. Fitting of experimental tandem dark $J-V$ characteristics of a single $a-\mathrm{Si}: \mathrm{H} \quad p-i-n$ cell, a single $\mu c-\mathrm{Si}: \mathrm{H} p-i-n$ cell, and of a tandem $a-\mathrm{Si}: \mathrm{H} / \mu c-\mathrm{Si}: \mathrm{H}$. The device configuration of the tandem structures is as follows:

$\mathrm{Au} / \mathrm{ITO} / p-\mu c-\mathrm{Si} / i-a-\mathrm{Si} / n-a-\mathrm{Si} / n-\mu c-\mathrm{Si} / \mathrm{AB} / p-\mu c-\mathrm{Si} / i-\mu c-\mathrm{Si} / n-a-\mathrm{Si} / \mathrm{SS}$. The intrinsic layer thicknesses of the single $a-\mathrm{Si}: \mathrm{H}$ and $\mu c-\mathrm{Si}: \mathrm{H}$ cells are 500 and $1400 \mathrm{~nm}$, respectively. In the tandem cell the top and bottom subcell intrinsic layer thicknesses are 200 and $1500 \mathrm{~nm}$, respectively.

the second exponential region but we were a bit off in the high voltage region. A very thin defective intrinsic layer between the $\mu c$-Si doped layers was included in the TRJ junction. Playing with the electrical parameters of this TRJ intrinsic layer and with $F_{o}(\mathrm{~V} / \mathrm{cm})$ we tailored the thermal generation rate until we could reach a good fitting of the high forward region. The most appropriated parameters for the TRJ intrinsic layer resulted to be a thickness of $2 \mathrm{~nm}$, a DB density of $2.5 \times 10^{18} \mathrm{~cm}^{-3}$, and $F_{o}(\mathrm{~V} / \mathrm{cm})$ of $\sim 1.1$ $\times 10^{5} \mathrm{~V} / \mathrm{cm}$. The value of $F_{o}$ is very similar to the one obtained by Willemen. ${ }^{34}$ At the lowest forward voltages the predicted dark $J-V$ results are lower than the measured $J-V$ characteristics in both single junctions and in the tandem solar cells which we ascribe to the presence of shunt. The electrical parameters used in the top and in the bottom intrinsic layers were the ones already listed in Table I. Our final result is shown in Fig. 8. We would like to stress that there was no need of modifying the parameters obtained from fitting dark $J-V$ curves of the single $p-i-n$ devices to match the dark $J-V$ characteristic of the tandem $p-i-n / p-i-n$ structure.

\section{CONCLUSIONS}

The transport mechanisms controlling the dark $J-V$ characteristic of micromorph solar cells are a combination of the mechanisms present in $a-\mathrm{Si}: \mathrm{H}$ and $\mu c-\mathrm{Si}$ single $p-i-n$ devices in conjunction with thermal generation of e-h pairs taking place inside the TRJ. In the dark $J-V$ of the micromorph structure two regions with exponential dependences and a third region where the current grows more slowly can be identified. In the first exponential region present at low forward voltages the current is controlled by recombination through gap states in the $a-\mathrm{Si}: \mathrm{H}$ intrinsic layer. In the second exponential region present at intermediate voltages, the current is controlled by recombination through gap states in the top and in the bottom intrinsic layers and by diffusion of free carriers along the $a-\mathrm{Si}: \mathrm{H}$ intrinsic layer. The third region of the dark $J-V$ curve is controlled by diffusion of free carriers in the $\mu c$-Si intrinsic layer, electron SCLC in the $a-\mathrm{Si}: \mathrm{H}$ intrinsic layer, and by thermal generation of e-h pairs inside the TRJ structure. The high forward dark $J-V$ curve can be used as an alternative diagnosis tool to check the efficiency of the TRJ in recombining the electron and holes that are photogenerated in the top and bottom cells. The dark $J-V$ curve of a micromorph tandem cell prepared by HWCVD was successfully fitted within the voltage regions free from shunts.

\section{ACKNOWLEDGMENTS}

We highly appreciate the financial support of Agencia Nacional de Promoción Científica y Tecnológica and CONICET through the Projects PICT (Grant No. 200212523) and PIP (Grant No. 5286). We thank Robert Stolk and Karine van de Werf for solar cell preparation.

${ }^{1}$ R. E. I. Schropp and M. Zeman, Amorphous and Microcrystalline Silicon Solar Cells: Modeling, Materials and Device Technology (Kluwer, Dordrecht, 1998).

${ }^{2}$ J. Meier, S. Dubail, J. Cuperus, U. Kroll, R. Platz, P. Torres, J. A. Anna Selvan, P. Pernet, N. Beck, and N. Pellaton Vaucher, Ch. Hof, D. Fisher, H. Keppner, and A. Shah, J. Non-Cryst. Solids 227-230, 1250 (1998).

${ }^{3}$ H. Keppner, J. Meier, P. Torres, D. Fisher, and A. Shah, Appl. Phys. A: Mater. Sci. Process. 69, 169 (1999).

${ }^{4}$ J. Yang and S. Guha, Appl. Phys. Lett. 61, 2917 (1992).

${ }^{5}$ H. Matsuura, A. Matsuda, H. Okushi, and K. Tanaka, J. Appl. Phys. 58, 1578 (1985).

${ }^{6}$ S. Hegedus, N. Salzamn, and E. Fagen, J. Appl. Phys. 63, 5126 (1988).

${ }^{7}$ M.A.Kroon and R. A. C. M. M. van Swaaij, J. Appl. Phys. 90, 994 (2001).

${ }^{8}$ J. Deng, J. M. Pearce, R. J. Koval, V. Vlahos, R. W. Collins, and C. R. Wronski, Appl. Phys. Lett. 82, 3023 (2003).

${ }^{9}$ C. van Berkel, M. J. Powell, A. R. Franklin, and I. D. French, J. Appl. Phys. 73, 5264 (1993).

${ }^{10}$ S. J. Fonash, Solar Cell Device Physics (Academic, New York, 1981).

${ }^{11}$ K. Lips, C. Boehme, and W. Fuhs, J. Non-Cryst. Solids 338-340, 702 (2004).

${ }^{12}$ T. Brammer and H. Stiebig, Mater. Res. Soc. Symp. Proc. 715, 641 (2002).

${ }^{13}$ T. Brammer and H. Stiebig, J. Appl. Phys. 94, 1035 (2003).

${ }^{14}$ J. J. H. Strengers, F. A. Rubinelli, J. K. Rath, and R. E. I. Schropp, Thin Solid Films 501, 291 (2006).

${ }^{15}$ P. J. McElheny, J. K. Arch, H. S. Lin, and S. J. Fonash, J. Appl. Phys. 64, 1254 (1988).

${ }^{16}$ F. A. Rubinelli, J. K. Rath, and R. E. I. Schropp, J. Appl. Phys. 89, 4010 (2001).

${ }^{17}$ F. A. Rubinelli and R. E. I. Schropp, Proceedings of the Second World Conference on PVEC, Vienna, Austria, 1998 (unpublished), Vol. 1, p. 998.

${ }^{18}$ M. Vukadinovic, F. Smole, M. Topic, R. E. I. Schropp, and F. Rubinelli, J. Appl. Phys. 96, 7289 (2004).

${ }^{19}$ E. Klimovsky, J. K. Rath, R. E. I. Schropp, and F. A. Rubinelli, J. NonCryst. Solids 338-340, 686 (2004).

${ }^{20}$ E. Klimovsky, A. Sturiale, and F. A. Rubinelli, Thin Solid Films 515, 4826 (2007).

${ }^{21}$ F. Rubinelli, IEEE Trans. Electron Devices 39, 2584 (1992).

${ }^{22}$ J. Pla, M. Barrera, and F. A. Rubinelli, Semicond. Sci. Technol. 22, 1122 (2007).

${ }^{23}$ A. Sturiale and F. A. Rubinelli, Internal Report on Single HWCVD Single $a$-Si and $\mu c$-Si cells (INTEC, UNL, Argentina, 2006).

${ }^{24}$ F. A. Rubinelli, J. K. Arch, and S. J. Fonash, J. Appl. Phys. 72, 1621 (1992).

${ }^{25}$ F. A. Rubinelli, H. Lou, and C. R. Wronski, Philos. Mag. B 74, 407 (1996).

${ }^{26}$ I. Chen and S. Lee, J. Appl. Phys. 53, 1045 (1982).

${ }^{27}$ S. M. Sze, Physics of Semiconductor Devices (Wiley, New York, 1981).

${ }^{28} \mathrm{X}$. Xu, J. Yang, A. Banerjee, S. Guha, K. Vasanth, and S. Wagner, Appl. Phys. Lett. 67, 2323 (1995).

${ }^{29}$ K. D. Mackenzie, P. G. Le Comber, and W. E. Spear, Philos. Mag. B 46, 377 (1982).

${ }^{30}$ J. J. H. Strengers, "A combined experimental and computer simulation study of HWCVD $n-i-p$ microcrystalline silicon solar cells," M.A. thesis, Utrecht University, 2005.

${ }^{31}$ Marieke van Veen, "Tandem solar cell deposited using hot-wire chemical vapor deposition,” Ph.D. thesis, Utrecht University, 2003. 
${ }^{32}$ B. Yan, G. Yue, J. Yang, S. Guha, D. L. Williamson, D. Han, and Ch.-S. Jiang, Appl. Phys. Lett. 85, 1955 (2004).

${ }^{33}$ J. Y. Hou, J. K. Arch, S. J. Fonash, S. Wiedeman, and M. Bennett, Proceedings of the 22nd IEEE PVSC, USA, 1991, p. 1260.

${ }^{34} \mathrm{~J}$. A. Willemen, "Modeling of amorphous silicon single- and multijunction solar cells," Ph.D. thesis, Technische Universiteit Delft, 1998.

${ }^{35} \mathrm{~S}$. Bae and S. J. Fonash, Proceedings of the 1st WCPEC PVSC, Hawaii, 1994, p. 484.

${ }^{36}$ G. A. M. Hurkx, D. B. M. Klaassen, and M. P. G. Knuvers, IEEE Trans. Electron Devices 39, 331 (1992)

${ }^{37}$ G. Juska, J. Kocka, K. Arlauskas, and G. Jukonis, Solid State Commun. 75, 531 (1990)

${ }^{38}$ G. Vincent, A. Chantre, and D. Bois, J. Appl. Phys. 50, 5484 (1979).
${ }^{39}$ Q. Gu, E. A. Schiff, J.-B. Chevrier, and B. Equer, Phys. Rev. B 52, 5695 (1995).

${ }^{40}$ S. S. Hegedus, F. Kampas, and J. Xi, Appl. Phys. Lett. 67, 813 (1995).

${ }^{41}$ J. K. Arch and S. J. Fonash, Appl. Phys. Lett. 60, 757 (1992).

${ }^{42}$ R. L. Stolk, H. Li, C. H. M. Van Der Werf, and R. E. I. Schropp, Thin Solid Films 501, 256 (2006).

${ }^{43} \mathrm{H}$. Li, F. Rubinelli, J. Rath, and R. E. I. Schropp, 21st European Photovoltaic Solar Energy Conference and Exhibition, Dresden, Germany, 4-9 September 2006 (unpublished).

${ }^{44} \mathrm{H}$. Li, "Single and multijunction solar cells on flexible substrate with absorber layers made with hot-wire CVD," Ph.D. thesis, Utrecht University, 2007. 